\title{
High-Frequency Band Automatic Mode Recognition Using Deep Learning
}

\author{
Zhengjia Xu \\ Al Savvaris \\ Antonios Tsourdos \\ School of Aerospace, Transport and Manufacturing \\ Cranfield University \\ Milton Keynes, UK \\ Zhengjia.Xu@cranfield.ac.uk
}

\author{
Tareq Alawadi \\ Department of Electronics Engineering \\ College of Tech. Studies (CTS) \\ PAAET, KUWAIT \\ t.alawadi@paaet.edu.kw
}

\begin{abstract}
Communication in High-Frequency (HF) band allows for good-quality, low-cost, and long-distance data-link transmission over diverse landscapes in aerial communication systems. However, as limited frequency resources are allocated, HF band suffers from poor spectrum efficiency when the channel is congested with many users. To maintain the robustness of the data-link transmission, Automatic Link Establishment (ALE) is the worldwide standard for sustaining HF communication of voice, data, instant messaging, internet messaging, and image communications. Technologies, such as spectrum sensing, Dynamic Spectrum Access (DSA) are utilised in ALE with the primary step of automatic mode recognition based on cognitive radio. Conventional methods, such as Automatic Modulation Recognition (AMR) targets at the classification of single modulation, while modern communication systems require recognising multiple modes in combination of various number of tones, tone spacing, and tone interval.

In this study, an approach that features filling the gap using deep learning is proposed. By characterising the common in-use mode formats in HF range, investigation shows that spectrogram diagram varies significantly, which necessitates the accurate characterisation and classification of multiple communication modes. Specifically, Convolutional Neural Network (CNN or ConvNet) is adopted for classification. The dataset is collected through USRP N210 with GNU Radio simulation. By reconstructing the communication in selected modes, the mode formats are classified. The performance result of recognition accuracy is displayed with confusion matrix. The confident classification of spectral characteristics, as well as accurate estimation, are established for practical communication scenarios.
\end{abstract}

Index Terms-High Frequency, Automatic Mode Recognition, Deep Learning, Spectrum Sensing, Convolutional Neural Network

\section{INTRODUCTION}

With an increasing demand for making the most of restricted radio spectrum resources in HF band, being aware of surrounding environment with cognitive radio (CR) has raised tremendous attention. In aeronautics, High-Frequency (HF) communication is still irreplaceable for long range, airground-air systems, such as Air Traffic System (ATS) over the North Atlantic, weather and airline communication system, Aeronautical Mobile (Route) Service (AM(R)S) and Aeronautical Mobile (Off Route) Service (AM(OR)S) [1]. According to [1], the congestion in HF voice frequency bands covering the North Atlantic will become unable to accommodate the increase in airline volume along with the reduction of vertical separation in high-density airspace regions by 2020-2025.

For statistics allocations, the overall spectrum usage varies from $15 \%$ to $85 \%$ (as per Federal Communications Commission) and remains idle most of the time [2]. To solve the paradox between spectrum scarcity and fill the spectrum hole that is caused by spectrum allocation in the Software Defined Network (SDN), the Dynamic Spectrum Access (DSA) allows unlicensed secondary users to access idle or unoccupied spectrums based on opportunistic mechanisms. These mechanisms primarily have the following qualities: to regard each state of channels as an independent distributed Markov process [3], to connect critical aspects in dynamic spectrum with game theory (named OSAG) [4] [5] and decision-theoretic solutions [6]. Furthermore, to reduce or avoid interference with other devices, the paper [7] concludes several system architectures in both cooperative and non-cooperative way. However, all the architectures have the foundation of accurate detection of transmitters and evaluation of communication condition.

In order to improve communication quality and raise efficiency in HF frequency, the Automatic Link Establishment (ALE) aims at establishing robust link quality and complementing flexibility in communication by using Link Quality Analysis (LQA) to help reduce the linking time and bring throughput improvement [8]. One of the challenging tasks in ALE is estimating the channel idle condition and obtaining the channel occupancy information without a prior knowledge.

The spectrum sensing, aiming at detecting and evaluating spectral occupancy has been done by many researchers. The sensing method varies; such as Energy Detection [9], matched filtering detection [10], wideband sensing [11], and compressed sensing [12]. The detailed spectrum sensing issues and solutions are referred to at [13] and [14]. Furthermore, spectrum inference/ prediction is a promising technique of inferring the occupied/ free state of radio spectrum, and it can also be efficient for raising efficiency by capturing the relevant information about spectral evolution to identify spectrum holes [15].

The similar topic is Automatic Modulation Recognition 
(AMR), which is also known as Automatic Modulation Classification (AMC). Generally, two usual categories can be classified: Likelihood-Based (LB) and Feature-Based (FB) methods [16]. The core idea of the LB approach is comparing the likelihood ratio against a threshold, following the criteria of minimising the probability of false classification. FB takes features into consideration, such as the variance of the signal amplitude, phase, and frequency. In recent years, owing to substantial advances in machine learning, a number of machine learning based FB techniques are used for solving the feature recognition issues, such as Artificial Neural Network (ANN) in [17] and support vector machines (SVM) in [18]. Particularly, deep learning is also introduced to solve Spectral Correlation Function (SCF) in modulation classification [19], and the adaption of Convolutional Neural Network (CNN or ConvNet) is utilised for the modulation recognition [20] with other variant neural networks [21] and [22].

Nevertheless, modern communication does not strictly obey any particular patterns or modes. Using the database managed by Wiki and Wavecom, over 100 modes have been identified in HF band, and many of them are still inactive in civilian, amateur and military applications. In addition, some standards have diverse working modes for adapting several operations, which brings more variations in mode recognition. All of these factors add to the difficulty of blind mode recognition by AMR technology.

The purpose of this study is to identify and recognise the availability of spectrum in HF band without prior knowledge with deep learning and take modern communication protocols into consideration as well. The features of the communication modes will be analysed first. Next, the details of deep learning architecture, as well as dataset acquisition methods, will be introduced. The final experimental result will be presented to validate the effectiveness of such an approach.

\section{Analysis of Communication Mode}

Because of the overcrowded mode natures in HF communication, creating and analysing separate models of individual transmission mode contributes to a dramatic incrementation in algorithm complexity and engineering quantity. Therefore, we propose to simplify mode types through the image-presenting way. Based on the database of waterfall chart and spectrogram from Wiki, Wavecom, and Go2monitor, the spectral occupancy result can be presented and labelled following these major features: the number of tones, tone spacing/ shift, and tone interval.

\section{A. Number of Tones}

The number of tones is one of the most influential indices to distinguish mode types. It is also intuitively presented in the spectrogram diagram. Four examples of different tones are displayed: 2 tones mode for Radio Teletype (RTTY) Fig. 1(a), 4 tones mode for Contestia with $125 \mathrm{~Hz}$ bandwidth Fig. 1(b), 16 tones mode for MIL-STD-188-110 Appendix A Fig. 1(c), and 39 tones mode for STANAG-4197 Fig. 1(d).

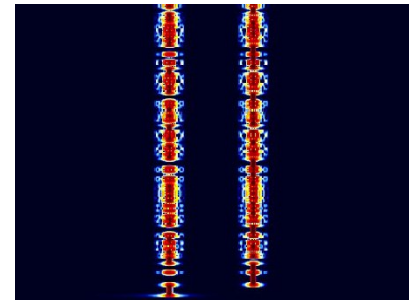

(a) RTTY

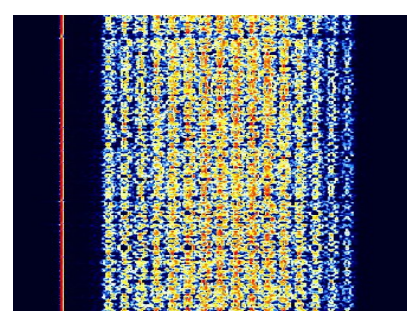

(c) MIL-STD-188-110

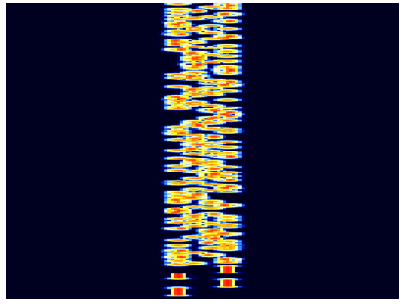

(b) Contestia-125

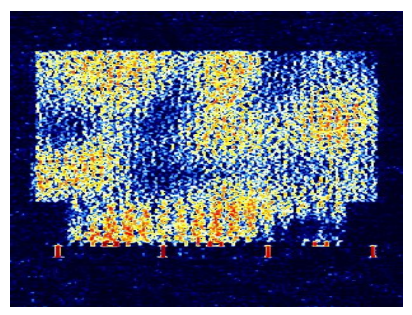

(d) STANAG-4197
Fig. 1. Sample Modes with Different Tone Numbers

\section{B. Tone Spacing}

Tone spacing, also known as shift in FSK, is the distance in frequency domain between each tone. It is important for this study because the burst communication between tone spacing is able to increase the efficiency. However, the recognition of tone spacing is difficult because the distance between tones, baud rate, interleave type, and transmitting power could contribute to the diversification of spectrogram diagram under the same mode, which ultimately may present difficulties for further classification.

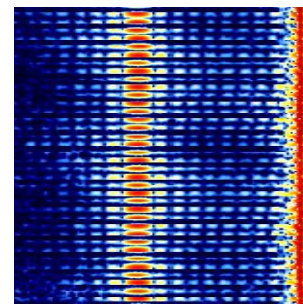

(a) Idle Mode
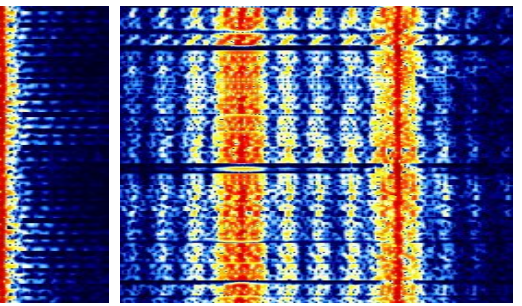

(b) Traffic Mode
Fig. 2. CIS with $400 \mathrm{~Hz}$ Shift Running at 96 Baud

In Fig. 2(a) and Fig. 2(b), the waterfall charts have significant variation with the same mode of CIS-14 $(650 \mathrm{~Hz}$ bandwidth with 2 FSK tones and $400 \mathrm{~Hz}$ shift), making the classification under such circumstance more challenging.

MIL-STD-188-110B-39-Tones standard has two interleaving coding means for enhancing the performance and robust quality. Fig. 3(a) and Fig. 3(b) indicate that the distinction in interleaving will also be apparent on spectrum.

\section{Tone Interval}

The tone interval is the scarcity in transmission, which is also meaningful for improving efficiency by burst communication between intervals.In some uncommon modes, the image of the waterfall chart does not appear to become continuous, 


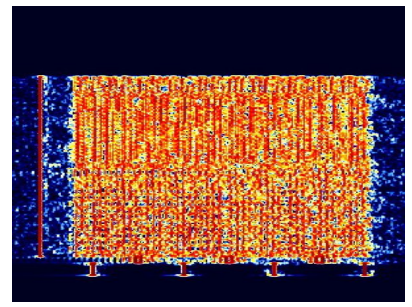

(a) Short Interleave

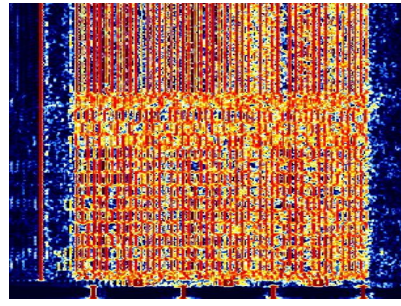

(b) Long Interleave
Fig. 3. Comparison of Two Interleaving in MIL-STD-188-110B

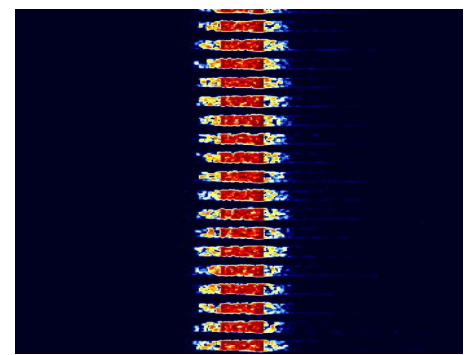

Fig. 4. Waterfall Chart of ARQ6-98 with $400 \mathrm{~Hz}$ Bandwidth

such as the ARQ6-98 mode in Fig. 4. An entire transmission cycle in ARQ6-98 has 490ms, including 210ms data block transmitting and $280 \mathrm{~ms}$ interval between data blocks, which has around $42.86 \%$ occupancy of bandwidth.

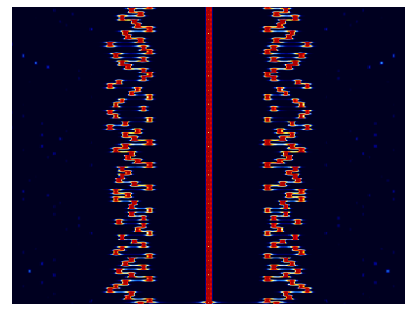

(a) $\mathrm{SP}-14$

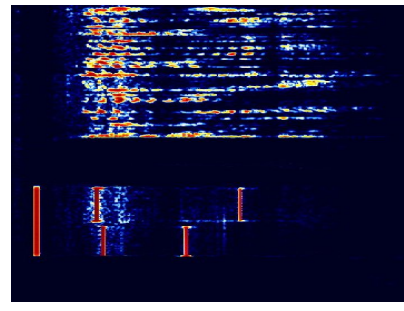

(b) ICAO Selcall
Fig. 5. Uncontinuous Transmission Modes

There are other unusual types of mode, such as SP-14 Fig. 5(a) and ICAO Selcal Fig. 5(b). To satisfy the robustness in transmission, these modes apply sufficient spectrum resource to ensure communication quality. Their spectrogram is intermittently hopping in multiple tones. Such special cases are not discussed in this study.

\section{System ARChitecture AND DATA ACQUisition}

For the purpose of training and testing, two separate datasets are required in the ConvNet network. In this paper the training dataset is simulated by software, and the testing dataset is acquired by hardware. The system architecture is illustrated in Fig. 6.

\section{A. ConvNet Network in Tensorflow}

We introduce the dataset into ConvNet network in image format, which converts the recognition of spectral occupancy into the issue of image pattern recognition. Some researches

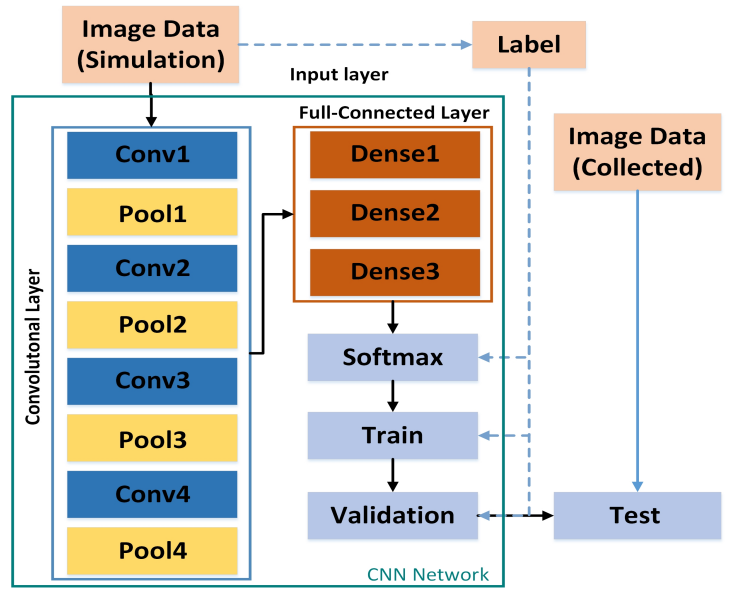

Fig. 6. Automatic Mode Recognition System Flow Diagram

have already been done with ConvNet network to solve pattern recognition by image processing and other architectures with the basis of ConvNet framework have proven to be efficient [23]. Therefore, due to the quality of simple function and small sample quantity, we adopted the ConvNet as the classifier as displayed in Fig. 6.

Using batch preprocessing procedure with the Open CV library, the corresponding labels can been generated. The original data is imported as image format at the size of $100 * 100$ pixels with 3 channels. According to Fig. 6 , the first convolutional layer Conv1 has 32 filters with $5 * 5$ kernel size and Rectified Linear Unit (ReLU) is employed as activation for accelerating the training speed [24]. The random initialisation of kernels has a distribution of 0.01 standard deviation. The first pooling layer Pool1 utilises Max Pooling algorithm with the pooling size of $2 * 2$ and the stride of 2 . The compressed image size after Pool1 layer turns to $50 * 50$ in pixel. The only distinctions between the first layer and the others are the filter number and kernel size. The image dimension converts to $25 * 25$ after Pool 2 layer and $12 * 12$ after Pool3 layer. After enduring the entire convolutional layer, the image size is $6^{*} 6$, and is ultimately reshaped for the input of full-connected layer.

The 3 dense layers have 1024, 512, and 12 units and the former 2 dense layers have ReLU for activation. The output size of neural units after dense layer is 12 . The loss function employs cross-entropy model embedded in Tensorflow. The simulation data with its label is also imported at this stage for the training and validation. The ConvNet network is saved and then restored for testing with the other dataset collected by hardware.

\section{B. Training Dataset Acquisition with GNU Radio}

For the reconstruction of diverse transmission modes, a large scale of mode patterns with the number of tones and tone spacing as labels is required. The samples are obtained by simulation with GNU Radio, and displayed with the waterfall chart module. The simulation framework of generating 2 tones is presented in Fig. 7. The digital information is 


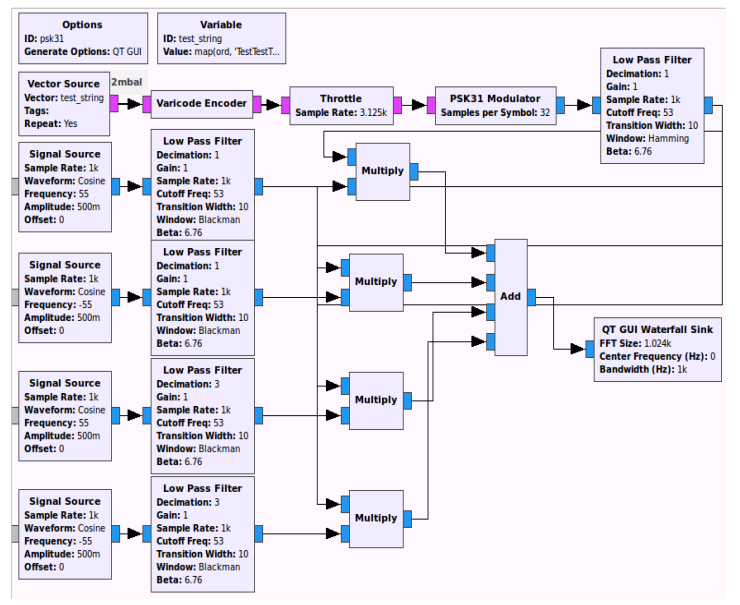

Fig. 7. GRC Simulation Diagram

imported for encoding. We chose the appropriate modulation and implemented a low-pass-filter (LPF) to eliminate the out-of-range bandwidth caused by digital modulation. The communication in multiple tones can be accomplished by multiplying the signal source and other carrier sources. At the same time, carrier sources should be connected to an LPF before multiplication as well. Finally, we merged several signals into one by the add module and utilised QT waterfall sink to display the simulation.

\section{Test Dataset Acquisition with USRP N210}
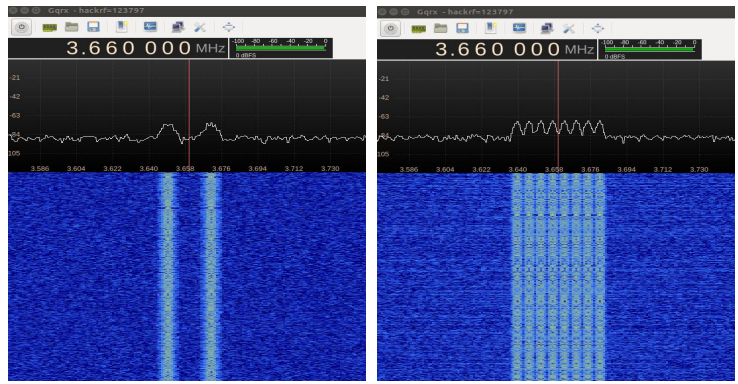

Fig. 8. Test Dataset Acquisition by Hardware

As one of the software methods for communication, SDR possesses light-development and flexible programming characteristics and it is preferred to other methods because it can accomplish diverse communication modes. The experiment hardware platform is implemented on the Universal Software Radio Peripheral(USRP) N210 embedded with LFTX and LFRX as daughterboards. The daughterboards are designed explicitly for $\mathrm{HF}$ transmission, which enables spectrum frequency ranging from $\mathrm{DC}$ to $30 \mathrm{MHz}$ covering entire $\mathrm{HF}$ band. The software development platform is a GNU radio with USRP Hardware Driver (UHD) as its interface, which has a processing framework comparable to the one that is displayed in Fig. 7. The spectrum collection is performed by using another SDR hardware HackRF One. The data acquisition result are presented in Fig. 8.

\section{RESUlTS AND COMPARISON}

In this study, the recognition of tone number and tone spacing is considered. The simulation has 2,4 and 8 tones, and 3 classes of tone spacing (narrow, medium, and wide). Therefore, the overall label types occupy $3 * 3$ groups. Meanwhile, the dataset collected by USRP must ensure its unicity and legality. The training samples are displayed in Fig. 9. 90\% simulation data is applied for training, and $10 \%$ for validation. The batchSize is set to 20 with the total training size of 386 . Another sample size acquired from hardware is the size of 360 for testing ConvNet network with 40 samples per group.

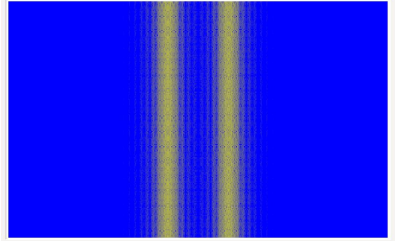

(a) Simulation of Training Sample (b) Simulation of Training Sample in 2 Tones with Wide Spacing

Fig. 9. Simulation of Tone Number

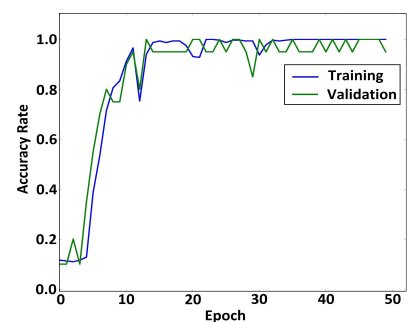

(a) Over Fitting

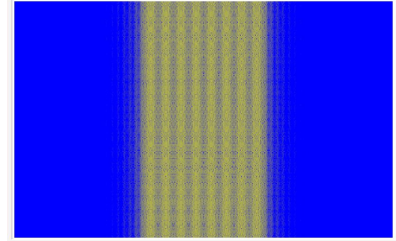

in 8 Tones with Narrow Spacing

\section{Fig. 10. ConvNet Accuracy Result}

It is noteworthy that the examples in Fig. 9 show a standard dataset with shortage of features, which is inappropriate for direct input to ConvNet. The lack of features in the samples will contribute to the over fitting issue, as explained in [25]. The over fitting result is shown in Fig. 10(a). As is apparent, both the training and validation results reach high accuracy since 16th epoch. However, in the testing phase, the result varies each time with approximately $30 \%$ accuracy. The over fitting problem can be resolved by adding noise, randomly cropping of image source, regularisation, and adjusting the ConvNet node number [25]. The results of optimised dataset and ConvNet network are presented in Fig. 10(b). The accuracy result climbs up to $80 \%$ at the 28th epoch. The training accuracy maintains at $99.997 \%$, and validation accuracy has the stable result around $97 \%$.

The test images were passed into the trained ConvNet network, and the confusion matrix result is shown in Fig. 11 with the average accuracy of $99.1802 \%$. The alphabetical representation in the figure follows the sequence combination of the 9 classes, such as narrow-2-tones for A, medium-2-tones for $\mathrm{B}$, wide-2-tones for $\mathrm{C}$ and so on. The result shows high 


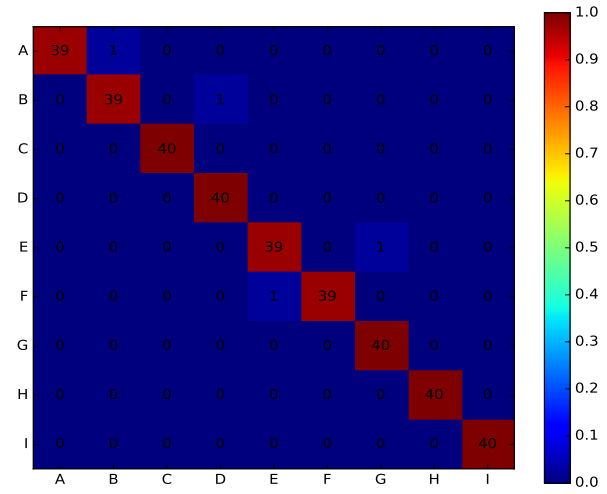

Fig. 11. ConvNet Test Accuracy Result

accuracy in classifying multiple communication modes and the ConvNet network is effective in automatic mode classification.

\section{CONCLUSION}

To recognise the spectral occupancy and increase the transmission efficiency in HF band, we analyse the modern protocols and modes, and conclude three principal features that are generally considered: tone number, tone spacing and tone interval. After converting the mode classification issue into image pattern classification problem, we employ deep learning for the classification by adopting the ConvNet network. The training data for ConvNet is generated by simulation, while the testing data is acquired by the hardware. The experiment result confirms the occupancy detection can be achieved by pattern recognition in image presenting way and the deep learning can be utilised in classifying communication modes.

However, it is necessary for more work to be undertaken. In this paper, we ignore the tone interval, which means we assume that the transmission is continuously made. Therefore, the uncontinuous transmission pattern will be simulated and validated afterwards. It is a benchmark and more neural network frameworks, such as Recurrent Neural Network (RNN) [26], have yet to be verified. There is a gap between classification and real-time recognition as well.

\section{REFERENCES}

[1] Jacob, Ponnu, et al. "Cognitive radio for aeronautical communications: A survey." IEEE Access 4 (2016): 3417-3443.

[2] I. F. Akyildiz, W. Y. Lee, M. C. Vuran, and S. Mohanty, "NeXt generation/dynamic spectrum access/cognitive radio wireless networks: A survey," In Computer Networks, vol. 50, no. 13, pp. 2127-2159, 2006.

[3] Altrad, Omar, et al. "Opportunistic spectrum access in cognitive radio networks under imperfect spectrum sensing." IEEE Transactions on Vehicular Technology 63.2 (2014): 920-925.

[4] Ji, Zhu, and KJ Ray Liu. "Cognitive radios for dynamic spectrum access-dynamic spectrum sharing: A game theoretical overview." IEEE Communications Magazine 45.5 (2007).

[5] Xu, Li, He Fang, and Zhiwei Lin. "Evolutionarily stable opportunistic spectrum access in cognitive radio networks." IET Communications 10.17 (2016): 2290-2299.

[6] Xu, Yuhua, et al. "Decision-theoretic distributed channel selection for opportunistic spectrum access: Strategies, challenges and solutions." IEEE Communications Surveys \& Tutorials 15.4 (2013): 1689-1713.

[7] McHenry, Mark Allen, et al. "Method and system for dynamic spectrum access." U.S. Patent No. 9,538,388. 3 Jan. 2017.
[8] L.Yu, J.Chen, G.Ding and Z.Qin, "Fast automatic link establishment: A new metric and the value of spectrum prediction," 2016 8th International Conference on Wireless Communications \& Signal Processing (WCSP), Yangzhou, 2016, pp. 1-6. doi: 10.1109/WCSP.2016.7752645.

[9] Cabric, Danijela, Artem Tkachenko, and Robert W. Brodersen. "Experimental study of spectrum sensing based on energy detection and network cooperation." Proceedings of the first international workshop on Technology and policy for accessing spectrum. ACM, 2006.

[10] Shobana, S., R. Saravanan, and R. Muthaiah. "Matched filter based spectrum sensing on cognitive radio for OFDM WLANs." International Journal of Engineering and Technology 5.1 (2013): 142-146.

[11] Sun, Hongiian, et al. "Wideband spectrum sensing for cognitive radio networks: a survey." IEEE Wireless Communications 20.2 (2013): 74 81.

[12] Tian, Zhi, and Georgios B. Giannakis. "Compressed sensing for wideband cognitive radios." Acoustics, Speech and Signal Processing, 2007. ICASSP 2007. IEEE International Conference on. Vol. 4. IEEE, 2007.

[13] Zeng, Yonghong, et al. "A review on spectrum sensing for cognitive radio: challenges and solutions." EURASIP Journal on Advances in Signal Processing 2010.1 (2010): 381465.

[14] Yucek, Tevfik, and Huseyin Arslan. "A survey of spectrum sensing algorithms for cognitive radio applications." IEEE communications surveys \& tutorials 11.1 (2009): 116-130.

[15] Ding, Guoru, et al. "Spectrum inference in cognitive radio networks: Algorithms and applications." IEEE Communications Surveys \& Tutorials (2017).

[16] Dobre, Octavia A., et al. "Survey of automatic modulation classification techniques: classical approaches and new trends." IET communications 1.2 (2007): 137-156.

[17] Kremer, Stefan C., and Joanne Shiels. "A testbed for automatic modulation recognition using artificial neural networks." Electrical and Computer Engineering, 1997. Engineering Innovation: Voyage of Discovery. IEEE 1997 Canadian Conference on. Vol. 1. IEEE, 1997.

[18] Petrova, Marina, Petri Mähönen, and Alfredo Osuna. "Multi-class classification of analog and digital signals in cognitive radios using Support Vector Machines." Wireless Communication Systems (ISWCS), 2010 7th International Symposium on. IEEE, 2010.

[19] Mendis, Gihan J., Jin Wei, and Arjuna Madanayake. "Deep learningbased automated modulation classification for cognitive radio." Communication Systems (ICCS), 2016 IEEE International Conference on. IEEE, 2016.

[20] O'Shea, Timothy J., Johnathan Corgan, and T. Charles Clancy. "Convolutional radio modulation recognition networks." International Conference on Engineering Applications of Neural Networks. Springer, Cham, 2016.

[21] Tang, Bin, et al. "Digital Signal Modulation Classification With Data Augmentation Using Generative Adversarial Nets in Cognitive Radio Networks.” IEEE ACCESS 6 (2018): 15713-15722.

[22] Zhang, Duona, et al. "Automatic Modulation Classification Based on Deep Learning for Unmanned Aerial Vehicles." Sensors 18.3 (2018): 924.

[23] Schmidhuber, Jürgen. "Deep learning in neural networks: An overview." Neural networks 61 (2015): 85-117.

[24] Krizhevsky A, Sutskever I, Hinton G E. Imagenet classification with deep convolutional neural networks[C]//Advances in neural information processing systems. 2012: 1097-1105.

[25] Xie, Lingxi, et al. "Disturblabel: Regularizing cnn on the loss layer." Proceedings of the IEEE Conference on Computer Vision and Pattern Recognition. 2016.

[26] Wang, Jiang, et al. "Cnn-rnn: A unified framework for multi-label image classification." Computer Vision and Pattern Recognition (CVPR), 2016 IEEE Conference on. IEEE, 2016. 
2018-12-10

\section{High-frequency band automatic mode recognition using deep learning}

Xu, Zhengjia

IEEE

Zhengjia Xu, Al Savvaris, Antonios Tsourdos and Tareq Alawadi. High-frequency band automatic mode recognition using deep learning. Proceedings of the 2018 IEEE/AIAA 37th Digital Avionics Systems Conference (DASC), 23-27 September 2018, London, UK. http://doi.org/10.1109/DASC.2018.8569367

Downloaded from Cranfield Library Services E-Repository 